\title{
Strong field limit analysis of gravitational retro-lensing
}

\author{
Ernesto F. Eiroa ${ }^{1, *}$, and Diego F. Torres ${ }^{2, \dagger}$ \\ ${ }^{1}$ Instituto de Astronomía y Física del Espacio, C.C. 67, Suc. 28, 1428, Buenos Aires, Argentina \\ ${ }^{2}$ Lawrence Livermore National Laboratory, 7000 East Ave., L-413, Livermore, CA 94550, USA
}

November 9, 2018

\begin{abstract}
We present a complete treatment in the strong field limit of gravitational retro-lensing by a static spherically symmetric compact object having a photon sphere. The results are compared with those corresponding to ordinary lensing in similar strong field situations. As examples of application of the formalism, a supermassive black hole at the galactic center and a stellar mass black hole in the galactic halo are studied as retro-lenses, in both cases using Schwarzschild and Reissner-Nordström geometries.
\end{abstract}

PACS numbers: 95.30.Sf, 04.70.Bw, 98.62.Sb

Keywords: General relativity, Black holes, Gravitational lensing

\section{Introduction}

Gravitational lensing is an important tool in astrophysics. Observable phenomena of lensing by stars and galaxies can be explained in the weak field approximation, that is to say, keeping only the first order term in the expansion of the deflection angle [1]. But when the lens is a black hole, a full general relativistic treatment is in order.

Recently, several articles studying strong field lensing appeared in the literature. Virbhadra \& Ellis 2] numerically analyzed the situation where the lens is a Schwarzschild black hole placed in the center of the Galaxy. They obtained the lens equation with an asymptotically flat metric and found that, besides the primary and secondary images, two infinite sets of faint relativistic images are formed by photons that make complete turns (in both directions of rotation) around the black hole before reaching the observer. Fritelli, Kling \& Newman [3] found an exact lens equation without any reference to a background metric and compared their results with those of Virbhadra \& Ellis. Bozza et al 44 developed an approximate analytical method (generally called, as well, the strong field limit) by which they obtained the positions and magnifications of the relativistic images for the Schwarzschild black hole. Subsequently, Eiroa, Romero \& Torres [5] applied the strong field limit to the Reissner-Nordström black hole and discussed the possibility of detection of the relativistic images in the next few years. Bozza [6] extended the strong field limit to any static spherically symmetric lens and analyzed the case of a charged Brans-Dicke black hole. Bhadra [7] studied the Gibbons-Maeda-Grafinkle-HorowitzStrominger charged black hole of string theory in the strong field limit. Virbhadra \& Ellis numerically investigated the lensing by naked singularities [8]. Bozza \& Mancini [9] used the strong field limit to

\footnotetext{
*e-mail: eiroa@iafe.uba.ar

†e-mail: dtorres@igpp.ucllnl.org
} 
study the time delay between different relativistic images, showing that different types of black holes are characterized by different time delays. Spinning black holes are more difficult to tackle. Bozza [10] analyzed the case of quasi equatorial lensing by rotating black holes and Vázquez \& Esteban [11] studied the Kerr black hole where the observer and source have arbitrary inclinations. Many of these recent advances (if not all) deal with theoretical implications of General Relativity in a regime which is well beyond our current technological capabilities for detection. Henceforth, the emphasis of these studies is not as much in predicting new phenomena that could be tested in a short period of time as it is in gaining insight on how gravitational lensing in particular, and gravity in general, behave in the strong field regimes.

In ordinary lensing situations, the lens is placed between the source and the observer. But if the lens is a compact object with a photon sphere and the observer is placed between the source and the lens, it leads to the formation of images with deflection angles closer to odd multiples of $\pi$. The observer sees the images in front and the source behind. This situation is called retro-lensing and was studied for the first time by Holtz \& Wheeler [12, who analyzed only the two stronger images for a black hole placed in the galactic bulge with the sun as source. They also proposed retro-lensing as a new mechanism for searching black holes. A similar idea was suggested in the context of defocusing gravitational lensing by Capozziello et al [13. De Paolis et al [14 recently analyzed the retro-lensing scenario for a bright star close to the massive black hole at the galactic center.

In this paper we give a complete treatment of retro-lensing by a static, spherically symmetric lens, using the strong field limit. In Section 2 we obtain the lens equation for retro-lensing and the deflection angle in the strong field limit. In Section 3, the position and magnification of the images are calculated. In Section 4, the results are compared with those for ordinary lensing and astrophysical examples are given in Section 5. Final concluding remarks are given in Section 6. Throughout the paper we use units such that $G=c=1$.

\section{Lens equation and deflection angle}

Consider an observer (o) placed between a point source of light (s) and a strong field object with a photon sphere (e.g. a black hole), which we will call the lens (l). The line joining the observer and the lens define the optical axis. The background space-time is considered asymptotically flat, with the observer and the source immersed in the flat region. We call $\beta$ the angular position of the source and $\theta$ the angular position of the images (i) as seen by the observer. The lens situation is shown in Fig. 1. We can take $\beta>0$ without loosing generality. The lens equation for retro-lensing is slightly different from ordinary lensing (i.e. when the lens lies between the source and the observer), due to the different relative positions of the lens, source and observer:

$$
\tan \beta=\tan \theta-\frac{d_{o s}}{d_{l s}}[\tan (\alpha-\theta)+\tan \theta]
$$

where $d_{o s}=D_{o s} / 2 M$ and $d_{l s}=D_{l s} / 2 M$ are, respectively, the observer-source and the lens-source distances in units of the Schwarzschild radius, and $\alpha$ is the deflection angle. In lensing situations where the objects are highly aligned, the angles $\beta$ and $\theta$ are small and $\alpha$ is closer to an odd multiple of $\pi$. For the images at the opposite side of the source (see Fig. 1), it can be written as $\alpha=(2 n-1) \pi+\Delta \alpha_{n}$, with $n \in \mathbb{N}$ and $0<\Delta \alpha_{n} \ll 1$. Then, the lens equation takes the form

$$
\beta=\theta-\frac{d_{o s}}{d_{l s}} \Delta \alpha_{n}
$$




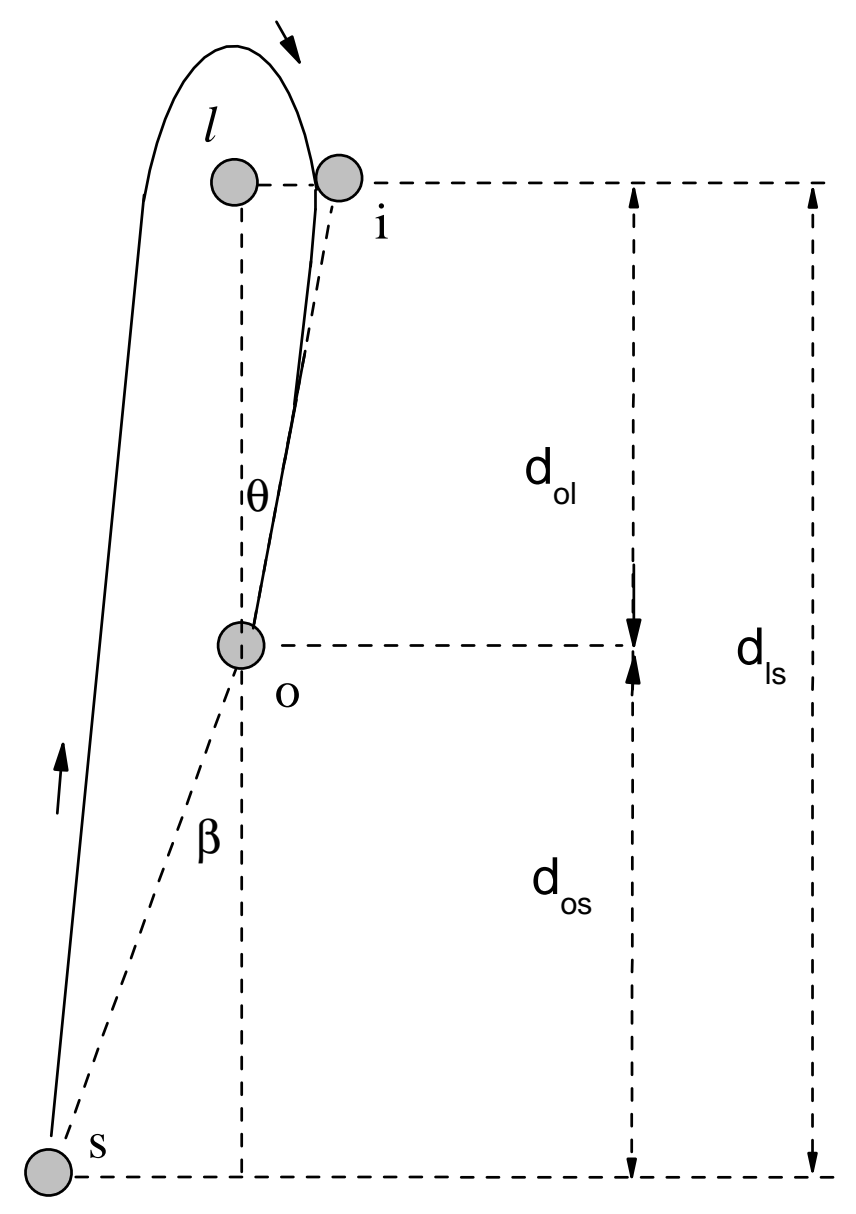

Figure 1: Lens diagram. The observer (o), the lens (l), the source (s) and the projection on the lens plane of the first relativistic image (i) are shown. The angular position of the source is $\beta$, of the image is $\theta$, and $d_{o l}, d_{o s}, d_{l s}$ are, respectively, the observer-lens, the observer-source and the lens-source distances. Two infinite sets of relativistic images (with both directions of rotation around the lens) are obtained with deflection angles close to odd multiples of $\pi$.

As in the case of ordinary strong field lensing, two infinite sets of relativistic images are formed. To obtain the other set of images (at the same side of the source) we should take $\alpha=-(2 n-1) \pi-\Delta \alpha_{n}$, so $\Delta \alpha_{n}$ must be replaced by $-\Delta \alpha_{n}$ in Eq. (2). In the case of perfect alignment, an infinite series of concentric Einstein rings are obtained.

To calculate the deflection angle, consider an static spherically symmetric metric

$$
d s^{2}=-f(x) d t^{2}+g(x) d x^{2}+h(x) d \Omega^{2},
$$

where $x=r / 2 M$ is the radial coordinate in units of the Schwarzschild radius. We require this metric to have a photon sphere, which radius $x_{p s}$ is given by the greater positive solution of the equation:

$$
\frac{h^{\prime}(x)}{h(x)}=\frac{f^{\prime}(x)}{f(x)}
$$


where the prime means derivative with respect to $x$.

The deflection angle corresponding to the images situated at the opposite side of the source as a function of the closest distance of approach $x_{0}$ is given by [15]

$$
\alpha\left(x_{0}\right)=I\left(x_{0}\right)-\pi
$$

with

$$
I\left(x_{0}\right)=\int_{x_{0}}^{\infty} 2\left(\frac{g(x)}{h(x)}\right)^{1 / 2}\left(\frac{h(x) f\left(x_{0}\right)}{h\left(x_{0}\right) f(x)}-1\right)^{-1 / 2} d x .
$$

The impact parameter $b$ (in units of the Schwarzschild radius) is [15]

$$
b\left(x_{0}\right)=\left(\frac{h\left(x_{0}\right)}{f\left(x_{0}\right)}\right)^{1 / 2}
$$

From the lens geometry we also have that

$$
b\left(x_{0}\right)=d_{o l} \sin \theta \approx d_{o l} \theta,
$$

with $d_{o l}=D_{o l} / 2 M$ the observer-lens distance in units of the Schwarzschild radius.

Bozza 6] has shown that for a spherically symmetric lens with a photon sphere, light rays with impact parameter $b$ close to the photon sphere have a deflection angle that can be approximated by

$$
\alpha=-a_{1} \ln \left(b-b_{p s}\right)+a_{2}+O\left(b-b_{p s}\right),
$$

where $b_{p s}=b\left(x_{p s}\right)$, and $a_{1,2}$ are constants which depend only on the type of lens. Provided the metric of the compact object that acts as a lens, the coefficients $a_{1,2}$ can be obtained in a laborious but straightforward way using the formalism developed in [6]. The method consists essentially in separating the integral of Eq. (6) in a divergent part which gives the logarithmic term in Eq. (9), and a regular part which gives the constant $a_{2}$. This approximation, named the strong field limit, gives very accurate results. For more details see [6].

Another way of calculating the constants $a_{1,2}$ is following a similar approach to that used by Eiroa, Romero \& Torres [5]:

$$
a_{1}=\lim _{x_{0} \rightarrow x_{p s}} \frac{-\left[b\left(x_{0}\right)-b\left(x_{p s}\right)\right] I^{\prime}\left(x_{0}\right)}{b^{\prime}\left(x_{0}\right)}
$$

and

$$
a_{2}=\ln \left\{\lim _{x_{0} \rightarrow x_{p s}}\left[e^{I\left(x_{0}\right)-\pi}\left[b\left(x_{0}\right)-b\left(x_{p s}\right)\right]^{a_{1}}\right]\right\},
$$

with the prime now meaning the derivative with respect to $x_{0}$. These limits can be evaluated numerically with standard software.

The values of the coefficients $a_{1}$ and $a_{2}$ for Schwarzschild and Reissner-Nordström lens geometries are given in the Appendix. 


\section{Position and magnification of the images}

Inverting Eq. (9) to obtain the impact parameter

$$
b=b_{p s}+\exp \left(\frac{a_{2}-\alpha}{a_{1}}\right)
$$

and using that $b=d_{o l} \theta$, we have that the position of any image is

$$
\theta=\frac{b_{p s}}{d_{o l}}+\frac{1}{d_{o l}} \exp \left(\frac{a_{2}-\alpha}{a_{1}}\right)
$$

Taylor expanding to first order around $\alpha=(2 n-1) \pi$, we can approximate the angular position of the $n$-th image by

$$
\theta_{n}=\theta_{n}^{0}-\frac{\xi_{n}}{d_{o l}} \Delta \alpha_{n}
$$

with

$$
\theta_{n}^{0}=\frac{b_{p s}}{d_{o l}}+\frac{1}{d_{o l}} \exp \left[\frac{a_{2}-(2 n-1) \pi}{a_{1}}\right]
$$

and

$$
\xi_{n}=\frac{1}{a_{1}} \exp \left[\frac{a_{2}-(2 n-1) \pi}{a_{1}}\right] \text {. }
$$

Then from Eq. (14) we obtain

$$
\Delta \alpha_{n}=-\frac{\theta_{n}-\theta_{n}^{0}}{\xi_{n}} d_{o l}
$$

and replacing it in the lens equation

$$
\beta=\theta_{n}+\frac{d_{o s} d_{o l}}{d_{l s} \xi_{n}}\left(\theta_{n}-\theta_{n}^{0}\right)
$$

The last equation can be written in the form

$$
\beta-\theta_{n}^{0}=\left(1+\frac{d_{o s} d_{o l}}{d_{l s} \xi_{n}}\right)\left(\theta_{n}-\theta_{n}^{0}\right)
$$

and as the second term in the parentheses is much greater than one, we have that

$$
\beta-\theta_{n}^{0} \approx \frac{d_{o s} d_{o l}}{d_{l s} \xi_{n}}\left(\theta_{n}-\theta_{n}^{0}\right)
$$

We finally obtain that the angular positions of the images are

$$
\theta_{n}=\theta_{n}^{0}+\frac{d_{l s} \xi_{n}}{d_{o s} d_{o l}}\left(\beta-\theta_{n}^{0}\right)
$$

The second term in Eq. (21) is a small correction on $\theta_{n}^{0}$, so all images lie very close to $\theta_{n}^{0}$. With a similar treatment, the other set of relativistic images have positions given by

$$
\theta_{n}=-\theta_{n}^{0}+\frac{d_{l s} \xi_{n}}{d_{o s} d_{o l}}\left(\beta+\theta_{n}^{0}\right)
$$

When $\beta=0$ an infinite sequence of Einstein rings is formed, with angular radius

$$
\theta_{n}^{E}=\theta_{n}^{0}-\frac{d_{l s} \xi_{n}}{d_{o s} d_{o l}} \theta_{n}^{0}
$$


Gravitational lensing conserves surface brightness, so the ratio of the solid angles subtended by the image and the source gives the amplification of the $n$-th image:

$$
\mu_{n}=\left|\frac{\sin \beta}{\sin \theta_{n}} \frac{d \beta}{d \theta_{n}}\right|^{-1} \approx\left|\frac{\beta}{\theta_{n}} \frac{d \beta}{d \theta_{n}}\right|^{-1} .
$$

Using Eq. (21) we have that

$$
\mu_{n}=\frac{1}{\beta}\left[\theta_{n}^{0}+\frac{d_{l s} \xi_{n}}{d_{o s} d_{o l}}\left(\beta-\theta_{n}^{0}\right)\right] \frac{d_{l s} \xi_{n}}{d_{o s} d_{o l}}
$$

which can be approximated to first order in $d_{l s} / d_{o s} d_{o l} \ll 1$ by

$$
\mu_{n}=\frac{1}{\beta} \frac{d_{l s}}{d_{o s} d_{o l}} \theta_{n}^{0} \xi_{n}
$$

The same result is obtained for the other set of images. The first relativistic image is the brightest one, and the magnifications decrease exponentially with $n$.

The total magnification, considering both sets of images, is $\mu=2 \sum_{n=1}^{\infty} \mu_{n}$, which can be written as

$$
\mu=\frac{2}{\beta} \frac{d_{l s} a_{3}}{d_{o s} d_{o l}^{2} a_{1}}
$$

where

$$
a_{3}=b_{p s} \frac{\exp \left(\frac{a_{2}-\pi}{a_{1}}\right)}{1-\exp \left(-\frac{2 \pi}{a_{1}}\right)}+\frac{\exp \left(\frac{2 a_{2}-2 \pi}{a_{1}}\right)}{1-\exp \left(-\frac{4 \pi}{a_{1}}\right)}
$$

\section{$\underline{\text { Extended sources }}$}

If the source is extended, we have to integrate over its luminosity profile to obtain the magnification:

$$
\mu=\frac{\iint_{S} \mathcal{I} \mu_{p} d S}{\iint_{S} \mathcal{I} d S}
$$

where $\mathcal{I}$ is the surface intensity distribution of the source and $\mu_{p}$ is the magnification corresponding to each point of the source. If the source is uniform, and we use polar coordinates $(R, \varphi)$ in the source plane, with $R=0$ in the optical axis, and we consider the source as a disk $D\left(R_{c}, R_{s}\right)$ of radius $R_{s}$ centered in $R_{c}$, the last equation takes the form

$$
\mu=\frac{\iint_{D\left(R_{c}, R_{s}\right)} \mu_{p} R d R d \varphi}{\pi R_{s}^{2}}
$$

and, using that $\beta=R / D_{\text {os }}$ is the angular position of each point of the source, we have that

$$
\mu=\frac{\iint_{D\left(\beta_{c}, \beta_{s}\right)} \mu_{p} \beta d \beta d \varphi}{\pi \beta_{s}^{2}},
$$

where $D\left(\beta_{c}, \beta_{s}\right)$ is the disk with angular radius $\beta_{s}$ centered in $\beta_{c}$. Then

$$
\mu_{n}=\frac{I}{\pi \beta_{s}^{2}} \frac{d_{l s}}{d_{o s} d_{o l}} \theta_{n}^{0} \xi_{n}
$$


and

$$
\mu=\frac{2 I}{\pi \beta_{s}^{2}} \frac{d_{l s} a_{3}}{d_{o s} d_{o l}^{2} a_{1}}
$$

with $I=\iint_{D\left(\beta_{c}, \beta_{s}\right)} d \beta d \varphi$. This integral can be calculated in terms of elliptic functions [4]:

$$
I=2 \operatorname{Sign}\left[\beta_{s}-\beta_{c}\right]\left[\left(\beta_{s}-\beta_{c}\right) E\left(\frac{\pi}{2}, \frac{-4 \beta_{s} \beta_{c}}{\left(\beta_{s}-\beta_{c}\right)^{2}}\right)+\left(\beta_{s}+\beta_{c}\right) F\left(\frac{\pi}{2}, \frac{-4 \beta_{s} \beta_{c}}{\left(\beta_{s}-\beta_{c}\right)^{2}}\right)\right],
$$

where

$$
\begin{gathered}
F\left(\phi_{0}, \lambda\right)=\int_{0}^{\phi_{0}}\left(1-\lambda \sin ^{2} \phi\right)^{-\frac{1}{2}} d \phi, \\
E\left(\phi_{0}, \lambda\right)=\int_{0}^{\phi_{0}}\left(1-\lambda \sin ^{2} \phi\right)^{\frac{1}{2}} d \phi,
\end{gathered}
$$

are respectively, elliptic integrals of the the first and second kind with the arguments $\phi_{0}$ and $\lambda$.

\section{Comparison with ordinary lensing}

In ordinary lensing the angular positions of the two sets of relativistic images, for a point source, are given by

$$
\theta_{n}=\theta_{n}^{0}+\frac{d_{o s} \xi_{n}}{d_{l s} d_{o l}}\left(\beta-\theta_{n}^{0}\right)
$$

and

$$
\theta_{n}=-\theta_{n}^{0}+\frac{d_{o s} \xi_{n}}{d_{l s} d_{o l}}\left(\beta+\theta_{n}^{0}\right)
$$

with

$$
\theta_{n}^{0}=\frac{b_{p s}}{d_{o l}}+\frac{1}{d_{o l}} \exp \left(\frac{a_{2}-2 n \pi}{a_{1}}\right)
$$

and

$$
\xi_{n}=\frac{1}{a_{1}} \exp \left(\frac{a_{2}-2 n \pi}{a_{1}}\right) .
$$

We see that for both cases (ordinary and retro-lensing), the images are formed close to $\theta_{n}^{0}$, which has values of the order of the angular radius of the photon sphere of the lens. From Eqs. (15) and (39) we observe that the images for retro-lensing lies a bit farther of the photon sphere than in the ordinary case.

The magnifications in ordinary lensing, for a point source, of the relativistic images are (both sets):

$$
\mu_{n}=\frac{1}{\beta} \frac{d_{o s}}{d_{l s} d_{o l}} \theta_{n}^{0} \xi_{n},
$$

and the total magnification, summing the magnifications of both sets of relativistic images, is

$$
\mu=\frac{2}{\beta} \frac{d_{o s} a_{3}}{d_{l s} d_{o l}^{2} a_{1}},
$$

where

$$
a_{3}=b_{p s} \frac{\exp \left(\frac{a_{2}-2 \pi}{a_{1}}\right)}{1-\exp \left(-\frac{2 \pi}{a_{1}}\right)}+\frac{\exp \left(\frac{2 a_{2}-4 \pi}{a_{1}}\right)}{1-\exp \left(-\frac{4 \pi}{a_{1}}\right)} .
$$


Comparing Eqs. (28) and (43) we see that the first term of $a_{3}$ in retro-lensing is greater by a factor $e^{\pi / a_{1}}$ and the second by a factor $e^{2 \pi / a_{1}}$ with respect to the ordinary case. Then, taking by example the lensing by a Schwarzschild black hole, and considering a situation where the factor involving the distances and the source angular position is the same, the magnification of the relativistic images in retro-lensing is greater by a factor about 24 compared with ordinary lensing.

In addition, with ordinary lensing, besides the two set of relativistic images, two weak field images with small deflection angles are produced by the lens. These weak field images do not appear in retrolensing, and it is the main difference in both cases. The weak field images are orders of magnitude stronger than the relativistic ones [4, 5] and lie very close to them. This makes detecting the relativistic images in ordinary lensing a very difficult task. In the case of retro-lensing, instead, the faint relativistic images are stronger and relatively easier to identify due to the absence of the brighter weak field images.

\section{$5 \quad$ Examples}

In this Section we apply the formalism to the Schwarzschild and Reissner-Nordström lens geometries. Two cases are analyzed: a super-massive black hole situated at the galactic center and a stellar mass black hole in the galactic halo. In both cases a nearby star is taken as source.

There is strong evidence of the existence of a super-massive black hole at the center of our galaxy [16]. We take a $M \sim 2.8 \times 10^{6} M_{\odot}$ black hole as an fiducial lens at a distance $D_{o l}=8.5 \mathrm{kpc}$. A star with radius $R=R_{\odot}$ situated at $D_{o s}=50 \mathrm{pc}$ is assumed as the source. In Table 1 the first (outer) Einstein ring angular position $\left(\theta_{1}^{E}\right)$ and the limiting angular value of the Einstein rings $\left(\theta_{\infty}^{E}\right)$ are given for the Schwarzschild and Reissner-Nordström black hole lenses. The images lie very close to the Einstein angular radii. For extended sources, when there is complete alignment, instead of a pair of images for each $n$ (one on each side), an annular shaped image is formed, with magnification $2 \mu_{n}$. The magnification of the first relativistic image and the total magnification, for perfect alignment, are also shown in Table 1 .

Table 1: Black hole in the galactic center as a gravitational retro-lens for a star situated at $50 \mathrm{pc}$ from the Earth. For several values of the charge $q$ (in units of the Schwarzschild radius), the angular radii of the photon sphere $\left(\theta_{p s}\right)$, the first Einstein ring $\left(\theta_{1}^{E}\right)$, and the limiting value of the Einstein rings $\left(\theta_{\infty}^{E}\right)$, are given. The magnifications, for perfect alignment and taking the source as extended with radius $R_{\odot}$, of the first image is $2 \mu_{1}$, whereas the total magnification is $\mu$.

\begin{tabular}{ccccccc}
\hline$|q|$ & 0 & 0.1 & 0.2 & 0.3 & 0.4 & 0.5 \\
\hline$\theta_{p s}(\mu \operatorname{arcsec})$ & 9.755 & 9.667 & 9.395 & 8.899 & 8.080 & 6.503 \\
$\theta_{1}^{E}(\mu$ arcsec $)$ & 17.39 & 17.28 & 16.95 & 16.37 & 15.53 & 15.04 \\
$\theta_{\infty}^{E}(\mu$ arcsec $)$ & 16.90 & 16.78 & 16.43 & 15.80 & 14.78 & 13.01 \\
$2 \mu_{1}\left(\times 10^{-10}\right)$ & 3.032 & 3.031 & 3.049 & 3.164 & 3.673 & 7.705 \\
$\mu\left(\times 10^{-10}\right)$ & 3.037 & 3.037 & 3.055 & 3.172 & 3.687 & 7.784 \\
\hline
\end{tabular}

Low mass black holes are common in our galaxy [17. Let us now consider a stellar mass black hole with mass $M=7 M_{\odot}$ in the galactic halo with $D_{o l}=4 \mathrm{kpc}$ as retro-lens and a star situated at $D_{o s}=50 \mathrm{pc}$ with radius $R=R_{\odot}$ as source. The results are given in Table 2 
Table 2: Same as Table 1 for a black hole in the galactic halo as a gravitational retro-lens with a star situated at $50 \mathrm{pc}$ from the Earth as source.

\begin{tabular}{ccccccc}
\hline$|q|$ & 0 & 0.1 & 0.2 & 0.3 & 0.4 & 0.5 \\
\hline$\theta_{p s}\left(\times 10^{-5} \mu\right.$ arcsec $)$ & 5.182 & 5.136 & 4.991 & 4.728 & 4.292 & 3.455 \\
$\theta_{1}^{E}\left(\times 10^{-5} \mu\right.$ arcsec $)$ & 9.236 & 9.178 & 9.002 & 8.696 & 8.249 & 7.990 \\
$\theta_{\infty}^{E}\left(\times 10^{-5} \mu\right.$ arcsec $)$ & 8.976 & 8.916 & 8.729 & 8.393 & 7.853 & 6.910 \\
$2 \mu_{1}\left(\times 10^{-21}\right)$ & 4.053 & 4.052 & 4.076 & 4.230 & 4.911 & 10.30 \\
$\mu\left(\times 10^{-21}\right)$ & 4.060 & 4.060 & 4.084 & 4.240 & 4.928 & 10.41 \\
\hline
\end{tabular}

In both examples, the dependence of the angular positions and magnifications of the images with the charge is weak, obtaining important changes only for extreme values of charge.

As the images lie close to the photon sphere, the angular positions of them take very small values, what increase with larger lens mass and smaller observer-lens distance. For the supermassive black hole at the galactic center, they are of the order of $10 \mu \mathrm{arcsec}$, and for the stellar mass black hole in the galactic halo they are about $10^{-5} \mu$ arcsec. Something similar happens with the magnifications, which also grow with greater lens mass and smaller observer-lens distance. For the supermassive black hole at the galactic center the magnifications are of order $10^{-10}$ and for the low mass one in the galactic halo of order $10^{-21}$.

As long as $d_{o s} \ll d_{o l}$, the angular positions and amplifications of the images are not very sensitive to the value of $d_{o s}$. So if we take the Sun or a star at $100 \mathrm{pc}$ from the Earth as source, the order of magnitude of the results shown in Tables 1 and 2 does not change. As the images appear highly demagnified, to improve the possibility of observing them, strong nearby sources with nearly perfect alignment are needed.

In all cases, the first image is the strongest one, carrying about $99 \%$ of the total luminosity.

\section{Concluding Remarks}

We have presented the strong field limit formalism for gravitational retrolensing. We have found the positions and magnifications of the relativistic images within the strong field limit, and studied some cases which might present particular interest and be a benchmark point for comparison with the normal lensing situation. As in the case of ordinary lensing, the relativistic images are faint. However, if we compare the magnification of retro-lensed relativistic images with those of the ordinary strong field lensing, we find that the former are significanlty stronger. Additionally, as no brighter weak field images appear in retro-lensing, it would be easier to detect them. The angular separation of the relativistic images is of course beyond the angular resolution of current or foreseeable technologies, and not only angular resolution but also sensitivity improvements are needed to detect them (see the discussion in Eiroa, Romero \& Torres [5]). The image separations and magnifications present values that make the observational identification of these relativistic images a challenge for the future: we are not aware of any current research, in any wavelength, which would allow to resolve the retro-lensing images in the next years. In the case of charged black holes, it is only for extreme values of charge that important changes are obtained. 


\section{Acknowledgements}

This work has been partially supported by UBA (UBACYT X-143, EFE). The work of DFT was performed under the auspices of the US DOE (NNSA), by UC's LLNL under contract No. W-7405Eng-48.

\section{Appendix: Values of the coefficients $a_{1}$ and $a_{2}$}

$\underline{\text { Schwarzschild black hole }}$

The metric functions are

$$
f(x)=g^{-1}(x)=1-\frac{1}{x}, \quad h(x)=x^{2},
$$

then

$$
\begin{gathered}
x_{p s}=\frac{3}{2}, \\
b\left(x_{0}\right)=x_{0}\left(1-\frac{1}{x_{0}}\right)^{-1 / 2}, \quad b_{p s}=\frac{3 \sqrt{3}}{2} .
\end{gathered}
$$

The coefficients can be calculated exactly [4, 6,

$$
\begin{gathered}
a_{1}=1 \\
a_{2}=\ln [324(7 \sqrt{3}-12)]-\pi .
\end{gathered}
$$

$\underline{\text { Reissner-Nordström black hole }}$

In this case

$$
f(x)=g^{-1}(x)=1-\frac{1}{x}+\frac{q^{2}}{x^{2}}, \quad h(x)=x^{2},
$$

where $q=Q / 2 M$ is the electric charge in units of the Schwarzschild radius. Then

$$
\begin{gathered}
x_{p s}=\frac{3}{4}\left[1+\left(1-\frac{32}{9} q^{2}\right)^{1 / 2}\right], \\
b\left(x_{0}\right)=x_{0}\left(1-\frac{1}{x_{0}}+\frac{q^{2}}{x^{2}}\right)^{-1 / 2}, \\
b_{p s}=\frac{\left(3+\sqrt{9-32 q^{2}}\right)^{2}}{4 \sqrt{2} \sqrt{3-8 q^{2}+\sqrt{9-32 q^{2}}}} .
\end{gathered}
$$

The coefficient $a_{1}$ can be calculated exactly [6]

$$
a_{1}=\frac{x_{p s} \sqrt{x_{p s}-2 q^{2}}}{\sqrt{\left(3-x_{p s}\right) x_{p s}^{2}-9 q^{2} x_{p s}+8 q^{4}}}
$$


and the coefficient $a_{2}$ can be approximated $[\underline{6}$ by

$$
a_{2}=c_{1}+c_{2}+a_{1} \ln b_{p s}-\pi
$$

with

$$
\begin{gathered}
c_{1}=2 \ln [6(2-\sqrt{3})]+\frac{8}{9}\{\sqrt{3}-4+\ln [6(2-\sqrt{3})]\} q^{2}+O\left(q^{4}\right), \\
c_{2}=a_{1} \ln \left[2\left(x_{p s}-q^{2}\right)^{2} \frac{\left(3-x_{p s}\right) x_{p s}^{2}-9 q^{2} x_{p s}+8 q^{4}}{\left(x_{p s}-2 q^{2}\right)^{3}\left(x_{p s}^{2}-x_{p s}+q^{2}\right)}\right] .
\end{gathered}
$$

\section{References}

[1] P. Schneider, J. Ehlers and E.E. Falco, Gravitational Lenses (Springer-Verlag, Berlin, 1992)

[2] K.S. Virbhadra and G.F.R. Ellis, Phys. Rev. D 62, 084003 (2000).

[3] S. Frittelli, T.P. Kling and E.T. Newman, Phys. Rev. D 61, 064021 (2000).

[4] V. Bozza, S. Capozzielo, G. Iovane and G. Scarpetta, Gen. Rel. Grav. 33, 1535 (2001).

[5] E.F. Eiroa, G.E. Romero and D.F. Torres, Phys. Rev. D 66, 024010 (2002).

[6] V. Bozza, Phys. Rev. D 66, 103001 (2002).

[7] A. Bhadra, Phys. Rev. D 67, 103009 (2003).

[8] K.S. Virbhadra and G.F.R. Ellis, Phys. Rev. D 65, 103004 (2002).

[9] V. Bozza and L. Mancini, arXiv:gr-qc/0305007 (2003).

[10] V. Bozza, Phys. Rev. D 67, 103006 (2003).

[11] S.E. Vázquez and E.P. Esteban, arXiv:gr-qc/0308023 (2003).

[12] D.E. Holtz and J.A. Wheeler, Astrophys. J. 578, 330 (2002).

[13] S. Capozziello, R. de Ritis, V.I. Mank'o, A.A. Marino and G. Marmo, Phys. Scripta 56, 212 (1997).

[14] F. De Paolis, A. Geralico, G. Ingrosso and A.A. Nucita, arXiv:astro-ph/0307493 (2003).

[15] S. Weinberg, Gravitation and Cosmology: Principles and Applications of the General Theory of Relativity (Wiley, New York, 1972).

[16] R. Schödel et al., Nature 419, 694 (2002)

[17] E. Algol and M. Kamionkowski, Mon. Not. Roy. Astron. Soc. 334, 553 (2002). 